\section{Sensing motion with tact}

When we handle an object, the relative movement between its surface and our skin provides the brain with valuable information about its characteristics. The existence of a subpopulation of neurons in the visual cortex that responds specifically to the direction of movement of a visual stimulus is widely known, but it remains unclear whether the perception of tactile motion relies on an analogous mechanism. Now, Pei et al. identify a population of neurons in the somatosensory cortex that encode the direction of a moving tactile stimulus regardless of its shape, its speed or the force with which it contacts the skin. This implies a strong analogy between visual and tactile motion processing.

The authors stimulated the fingertips of rhesus macaques in precise spatiotemporal sequences to create the tactile equivalent of pixels on a monitor. When the tactile stimulus was applied, different subpopulations of neurons in area 1 of the somatosensory cortex responded preferentially to specific directions of motion. Remarkably, the preferred direction of a given subpopulation was the same for different types of moving stimuli, including a solid bar, a regular pattern of dots, or displays of dots the motion of which varied in overall coherence (the dots moved in generally the same direction). Moreover, by examining responses from individual neurons in the macaques, the authors could discriminate between directions of movement of the stimulus with similar accuracy to human subjects receiving the same type of tactile stimulation. This suggests that the response of these neurons might underlie the ability to perceive the direction of tactile motion, although this remains to be shown in experiments in which the same individual provides both behavioural and electrophysiological data.

In vision, information about motion is encoded by specialized neuronal subpopulations at each stage of processing and, ascending the perceptual pathway, motion representations become increasingly independent of other object properties. The findings in this study suggest that this may be the general case in the development of motion representations, regardless of whether we experience the moving stimulus through our eyes or our fingertips.

Cristian Bodo

ORIGINAL RESEARCH PAPER Pei, Y.-C. et al.

Shape invariant coding of motion direction in somatosensory cortex. PLoS Biol. 8, e1000305 (2010)

FURTHER READING Johansson, R. S. \&

Flanagan, J. R. Coding and use of tactile signals from the fingertips in object manipulation tasks. Nature Rev. Neurosci. 10, 345-359 (2009)

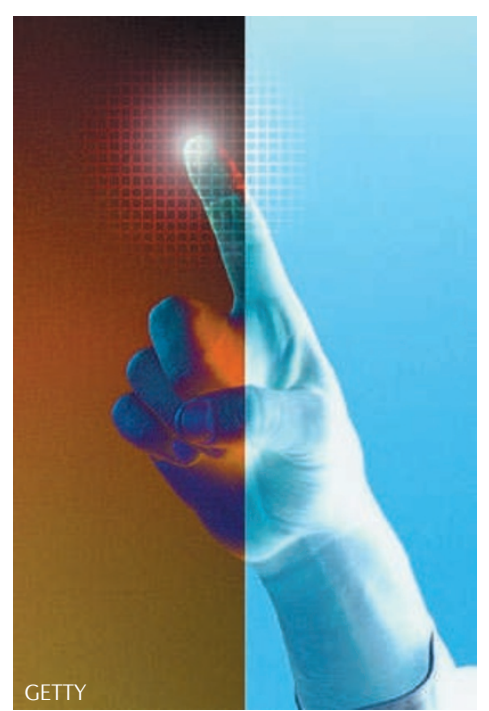

